\title{
Unrepeatered transmission reach extension by receiver-side all-optical back- propagation
}

Kaminski, P. M.; Da Ros, Francesco; Da Silva, E. P.; Clausen, A. T.; Forchhammer, Søren; Oxenløwe, Leif Katsuo; Galili, Michael

\section{Published in:}

Proceedings of 2019 24th OptoElectronics and Communications Conference

Link to article, DOI:

10.23919/PS.2019.8817641

Publication date:

2019

Document Version

Peer reviewed version

Link back to DTU Orbit

Citation (APA):

Kaminski, P. M., Da Ros, F., Da Silva, E. P., Clausen, A. T., Forchhammer, S., Oxenløwe, L. K., \& Galili, M. (2019). Unrepeatered transmission reach extension by receiver-side all-optical back-propagation. In Proceedings of 2019 24th OptoElectronics and Communications Conference IEEE. https://doi.org/10.23919/PS.2019.8817641

\section{General rights}

Copyright and moral rights for the publications made accessible in the public portal are retained by the authors and/or other copyright owners and it is a condition of accessing publications that users recognise and abide by the legal requirements associated with these rights.

- Users may download and print one copy of any publication from the public portal for the purpose of private study or research.

- You may not further distribute the material or use it for any profit-making activity or commercial gain

- You may freely distribute the URL identifying the publication in the public portal 


\title{
Unrepeatered Transmission Reach Extension by Receiver-Side all-Optical Back-Propagation
}

\author{
P.M. Kaminski ${ }^{1}$, F. Da Ros ${ }^{1}$, E.P. da Silva ${ }^{1,2}$, A.T. Clausen ${ }^{1}$, S. Forchhammer ${ }^{1}$, L.K. Oxenløwe ${ }^{1}$, M. Galili ${ }^{1}$ \\ 1. DTU Fotonik, Technical University of Denmark, DK-2800 Kgs. Lyngby, Denmark \\ 2. Electrical Engineering Department, Federal University of Campina Grande (UFCG), Campina Grande, Paraíba, Brazil \\ pkam@fotonik.dtu.dk
}

\begin{abstract}
Improvements in SNR (1.98 dB), Mutual Information (0.95 bit/symbol) and maximum transmission distance (almost $12 \%$ ) are demonstrated for dual-polarization WDM 16-QAM unrepeatered transmission using optical-phase-conjugation-based back-propagation at the receiver through power and dispersion engineering.

Keywords: Transmission impairments and their mitigations; Optical signal processing techniques for optical communications; Optical transmitter and receiver subsystems.
\end{abstract}

\section{INTRODUCTION}

Current optical communication systems are approaching the performance limit imposed by the inherent fiber nonlinearity, which currently constraints the achievable information rates and transmission distances [1]. Suppressing or compensating the nonlinearity has become a key requirement to increase overall system throughput and meet the future traffic demands. The competing techniques comprise digital and all-optical approaches. Digital methods typically introduce significant processing time, and are limited by the available receiver bandwidth, failing to effectively compensate for inter-channel nonlinearities in wavelength-division multiplexed (WDM) systems [1]. Alternatively, alloptical schemes can tackle the nonlinear distortions instantaneously across all the channels, and have therefore been attracting increasing attention. Among them, optical-phase conjugation (OPC) has a strong potential to reverse the impairments by mid-span spectral inversion (MSSI), and it can be easily implemented by means of four wave mixing (FWM). Despite the evident gains [1], for effective nonlinearity mitigation by MSSI power and dispersion symmetry with respect to the OPC device is required. This is both impractical with regard to the OPC position and challenging in terms of targeted power profiles. Moreover, the approach is incompatible with unrepeatered systems where no active devices are placed in the link.

In this work, we rely on the more fundamental criterion of equivalent pulse evolution on left- and right-hand sides of OPC instead of strictly symmetric profiles. We experimentally demonstrate nonlinearity compensation of unrepeatered transmission by all-optical back-propagation (OBP) at the receiver. The OBP module consists of an OPC device followed by a short nonlinear and dispersive medium, where the propagation closely resembles the nonlinear signal transmission. Efficient nonlinearity cancellation is achieved by proper dispersion engineering and nonlinear phase-shift optimization in the scaled-down structure. Aside from suppression of fiber nonlinearities, the scheme also provides optical dispersion compensation, potentially reducing the power consumption of the receiver digital signal processing (DSP) engine. The study is conducted for seven WDM 16-quadrature amplitude modulation (16-QAM) channels at 16 GBd. Numerical characterizations of ideal schemes of OPC-based OBP have previously been provided in [2,3]. However, these investigations have generally been based on setups challenging to implement in practical systems. A few experimental demonstrations have been reported for dispersion-managed links, but only focusing on optical pre-compensators at the transmitter side [4]. As the penalty associated with implementing OPC is higher for a high quality signal, as shown in [5], in this work we demonstrate significant gains by performing receiver-side compensation.

\section{PRinCiPle AND EXPERIMENTAl SETUP}
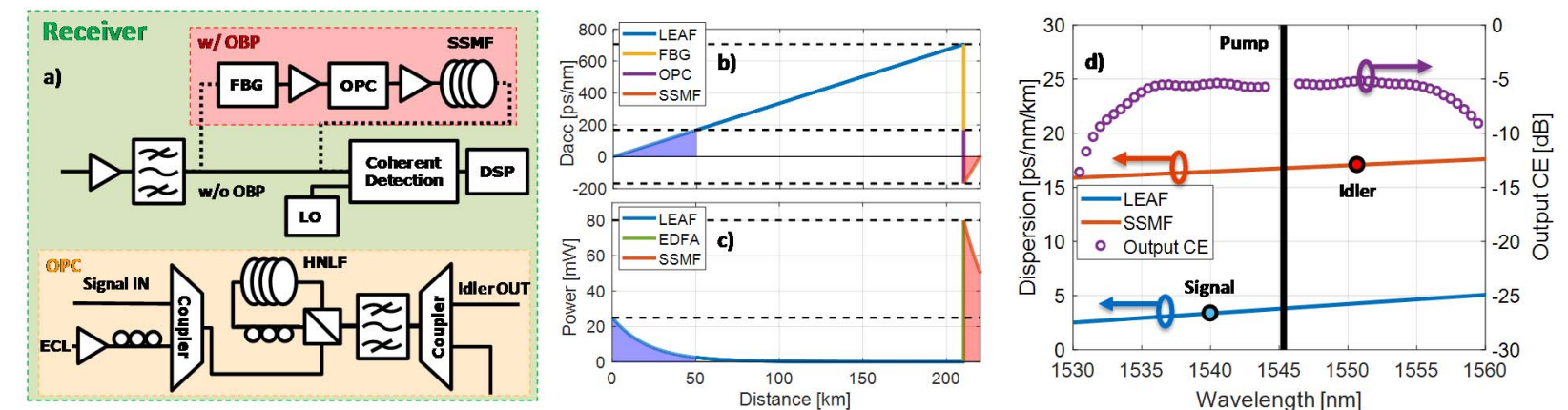

Fig. 1. (a) Schematics of OBP and OPC modules at the receiver. (b) Evolution of accumulated dispersion and (c) power versus distance with OBP module at the receiver included. (d) Dispersion characteristics of LEAF (blue) and SSMF (red) versus wavelength with signal, pump and idler position indicated, and output conversion efficiency of HNLF (purple). 
Efficient nonlinearity compensation by OBP is achieved only if the same nonlinear phase shifts $\Phi_{N L}=\gamma P(z, t) L$ occur on both sides of the OPC for identical pulse shapes of signal and idler (conjugated copy), respectively [6]. As shown in Fig. 1(a), the OBP module consists of three main components: a fiber Bragg grating (FBG) for dispersion compensation, OPC based on a strained highly nonlinear fiber (HNLF) in a polarization insensitive scheme [7], and $10 \mathrm{~km}$ of standard single-mode fiber (SSMF). The OPC setup operates under single pump-configuration, with an external cavity laser (ECL) pump at $1545.32 \mathrm{~nm}$ and $25 \mathrm{dBm}$ of pump power per polarization, resulting in an output conversion efficiency (CE) of $-6 \mathrm{~dB}$. WDM couplers facilitate coupling the pump with signal as well suppressing the pump at the OPC output. After conjugation, the idler (alone) is propagated through a scaled-down version of the transmission link (SSMF), which effectively mimics the MSSI approach, though with nonlinearity compensation in a more lumped fashion. The principle of operation is illustrated in Fig. 1(b)-(c), by considering power and dispersion evolutions. The signal is launched into $210 \mathrm{~km}$ of a non-zero dispersion-shifted (NZDS) large-effective area fiber (LEAF), and starts accumulating dispersion (Fig. 1b) and nonlinear phase shift (proportional to power: Fig. 1c) as it propagates. The transmission fiber choice was dictated by the low dispersion coefficient and enabled optical back-propagation at the receiver in a more dispersive, but shorter structure, which still accurately reproduces pulse evolution throughout the link. Throughout the unrepeatered transmission link, dispersion accumulates linearly with distance, while the nonlinear phase shift is dominant at the beginning of the link and quickly becomes negligible due to the exponential power decay. For this reason, the propagation beyond $50 \mathrm{~km}$ (nonlinear length) can be considered linear. Upon reaching the receiver, a FBG (approx. -560 ps/nm) [8] is used to compensate for the dispersion accumulated over the last $160 \mathrm{~km}$ of LEAF (total length - nonlinear length), restoring the pulse shape at $50 \mathrm{~km}$. Subsequently, the signal enters the OPC, where it is conjugated and wavelength shifted (Fig. 1d). Due to chirp reversal, the accumulated dispersion changes the sign. The conjugated idler then enters the SSMF for nonlinearity compensation. As depicted in Fig. 1(d), signal propagating through LEAF undergoes approx. $3.5 \mathrm{ps} / \mathrm{nm} / \mathrm{km}$ of dispersion, which increases to over $17 \mathrm{ps} / \mathrm{nm} / \mathrm{km}$ for the corresponding idler in SSMF. Dispersion in SSMF accumulates much faster in distance, and $10 \mathrm{~km}$ of propagation in SSMF corresponds to the first $50 \mathrm{~km}$ of LEAF, thus allowing to scale down the OBP compensation medium. As a result of the scaling, the nonlinear phase accumulation must be proportionately increased to keep up with rapidly changing pulse shapes. This can be controlled by adjusting the launched power into SSMF accordingly. Ultimately, with power into the SSMF optimized for a given launch power into the LEAF, the left- and right hand sides of the OPC are approximately equivalent in terms of dispersive and nonlinear pulse evolution. Blue and red shading in Fig. 1(b) and (c) denote similar nonlinearity at close to identical pulse shapes for LEAF and SSMF, respectively, which will be compensated for to a large degree. On top of that, complete all-optical dispersion compensation is achieved.

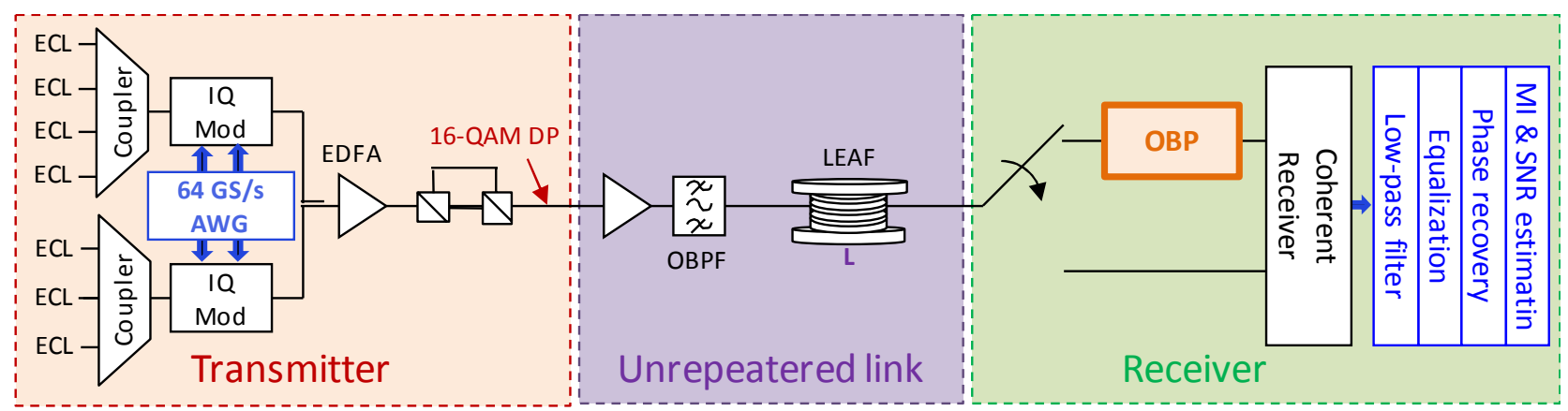

Fig. 2. Experimental setup for the transmission measurements with OPC-based optical back-propagation at the receiver side.

The experimental setup is sketched in Fig. 2. At the transmitter site, seven ECLs on a 25-GHz grid at $1540 \mathrm{~nm}$ are modulated by two IQ modulators (one for even and one for odd channels) with 16-QAM data at 16 GBd. All channels are coupled together and polarization multiplexing is emulated. At the transmitter output the signals are amplified, amplified spontaneous emission (ASE) noise is suppressed with an optical bandpass filter (OBPF), and they are injected directly into the unrepeatered transmission link. At the end of the transmission, the signal is pre-amplified, optically backpropagated and received with a coherent receiver followed by offline digital signal processing, including equalization and carrier phase recovery. The performance is then evaluated on a per-channel basis in terms of effective received signal-tonoise ratio (SNR) and mutual information (MI). MI is predicted based on Monte Carlo estimation and the effective received SNR is estimated directly from the transmitted $x_{k}$ and received $y_{k}$ QAM symbols after all DSP blocks according to: $S N R_{e f f}=\mathbb{E}_{k}\left[\left|x_{k}\right|^{2}\right] / \mathbb{E}_{k}\left[\left|y_{k}-x_{k}\right|^{2}\right]$ as in [9].

\section{EXPERIMENTAL RESULTS}

The performance after transmission is evaluated in terms of MI and SNR as functions of total signal launched power into the LEAF, and the cases with and without OBP are compared. For the OBP scenario, the input signal power into the OPC loop is kept at approx. $10 \mathrm{dBm}$, whereas the idler power injected into the SSMF compensation fiber is adjusted for each launch power. At the optimum launch power with OBP, the input to the SMF is approx. $19.5 \mathrm{dBm}$, i.e. $5 \mathrm{~dB}$ higher 
than in the LEAF. In the linear region, where the signal is greatly dominated by ASE noise after transmission, the OBP module introduces negligible degradation, as shown in Fig. 3(a)-(b). However, compared to the benchmark of 210-km transmission with no OBP, the optimum launch power shifts by approx. $2 \mathrm{~dB}$ and, more importantly, $1.8 \mathrm{~dB}$ of SNR improvement is achieved for the central channel, corresponding to $0.85 \mathrm{bit} / 2 \mathrm{D}$-symbol of MI gain. Considering a shorter link of $188 \mathrm{~km}$ without OBP, similar performance as for $210-\mathrm{km}$ with OBP is obtained, yielding almost $12 \%$ of reach extension. The performance improvement is consistent across all seven WDM channels with a mean SNR gain of $1.98 \mathrm{~dB}$ and mean MI improvement of 0.95 bit/2D-symbol, as shown in Fig. 3(c). Constellation diagrams in Fig. 3(d) at the optimum launch powers with and without OBP further outline the improved signal quality.
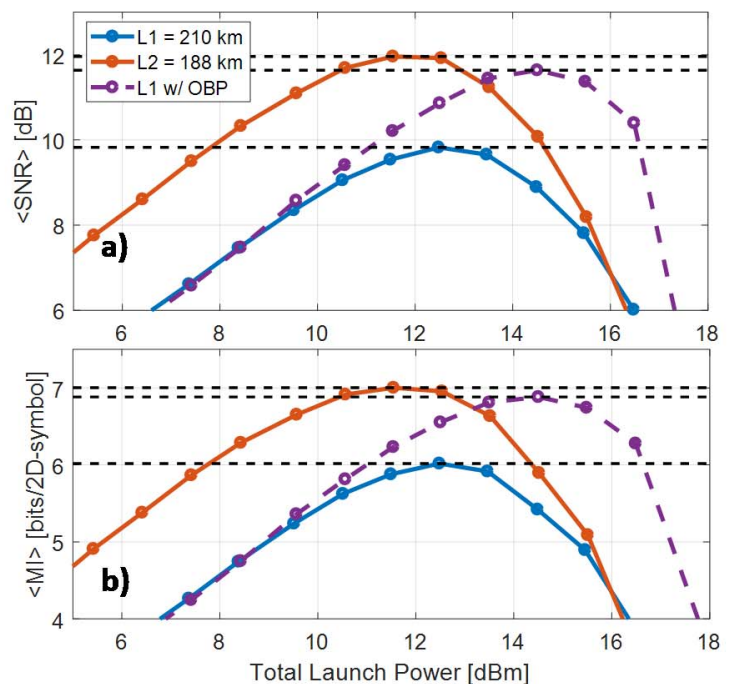
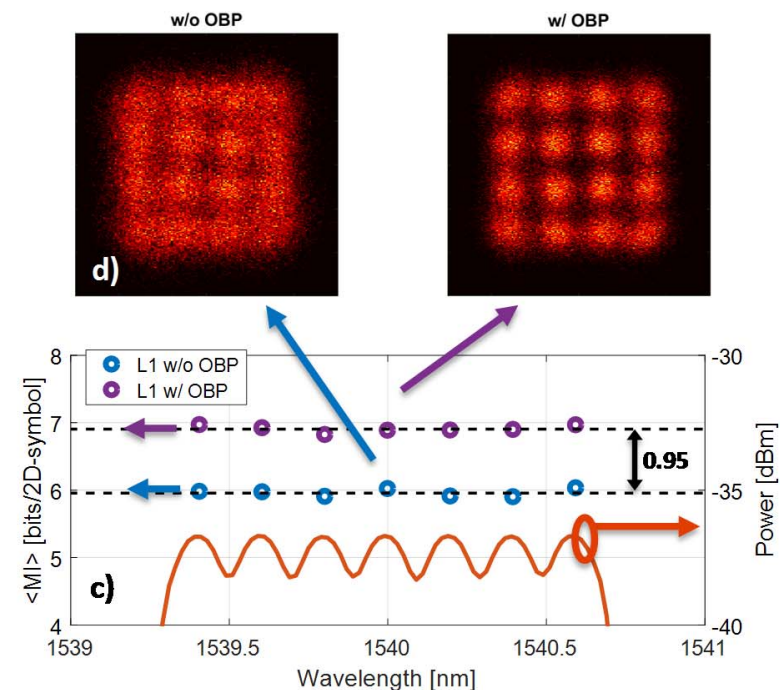

Fig. 3. Effective receiver SNR (a) and estimated MI (b) for the central channel without (blue) and with (purple) OBP for $210 \mathrm{~km}$ of straight transmission, and 188-km transmission without OBP (red). c) MI per channel without (blue) and with (purple) OBP. d) Corresponding constellation diagrams (X polarization only) at optimum launched power for the central channel.

\section{CONCLUSIONS}

We experimentally demonstrate a receiver-side all-optical back propagation scheme for efficient nonlinearity compensation for unrepeatered transmission. The OBP module consists of three main components: FBG for dispersion matching, polarization-insensitive OPC for chirp reversal, and short spool of SSMF for nonlinearity cancellation. The study shows average per channel improvement of $1.98 \mathrm{~dB}$ (SNR) and $0.95 \mathrm{bit} / 2 \mathrm{D}$-symbol (MI). Equivalently, implementing the OBP allows for almost $12 \%$ of reach extension without performance degradation. The principle demonstrated here can be applied also to Raman-amplified systems with the OBP adjusted to match the corresponding nonlinear regions, or transmission in SSMF links, given that a highly dispersive medium is available for the OBP.

\section{ACKNOWLEDGMENT}

\section{CoE SPOC (DNRF123), ERC CoG FRECOM (771878), OFS Denmark for HNLF, Proximion and KTH for FBG.}

\section{REFERENCES}

[1] A.D. Ellis et al., "Performance limits in optical communications due to fiber nonlinearity," Advances in Optics \& Photonics 9(3), 429-503 (2017).

[2] X. Liang et al., "Ideal optical backpropagation of scalar NLSE using dispersion-decreasing fibers for WDM transmission," Optics Express 21(23), 28668-28675 (2013).

[3] J. Shao et al., "Optical backpropagation for fiber-optic communications using optical phase conjugation at the receiver," Optics Letters 37(15), 3012-3014 (2012).

[4] M.D. Pelusi, "WDM Signal All-Optical Precompensation of Kerr Nonlinearity in Dispersion-Managed Fibers," IEEE Photonics Technology Letters, 25(1), 71-74 (2013).

[5] P.M. Kaminski et al., "Signal-to-Idler Conversion Penalty in AlGaAs-on-Insulator Wavelength Converter," in Proc. CLEO 2018, paper Stu4C.6.

[6] P. Minzioni, "Nonlinearity Compensation in a Fiber-Optic Link by Optical Phase Conjugation," Fiber and Integrated Optics 28(3), 179-209 (2009).

[7] F. Da Ros et al., "Link-Placement Characterization of Optical Phase Conjugation for Nonlinearity Compensation," in Proc. OFC 2018, paper W3E.3.

[8] O. Ozolins et al., "112 Gbps/ $\lambda$ PAM4 Inter-DCI with Continuous-Fiber Bragg Grating based Dispersion Compensators," in Proc. Advanced Photonics (2018), paper NeTh3F.3.

[9] M.P. Yankov et al., "Constellation shaping for WDM systems using 256QAM/1024QAM with probabilistic optimization," Journal of Lightwave Technology 34(22), 5146-5156 (2016). 